\title{
EVALUACIÓN POSTURAL DE INDIVIDUOS MAPUCHE DE LA ZONA COSTERA DE LA IX REGIÓN DE CHILE
}

\author{
POSTURAL EVALUATION OF MAPUCHE INDIVIDUALS IN THE COASTAL AREA OF THE IX REGION IN CHILE
}

\author{
Mariano del Sol \& Karina Hunter
}

DEL SOL, M. \& HUNTER, K. Evaluación postural de individuos mapuche de la zona costera de la IX Región de Chile. Int. J. Morphol., 22(4):339-342, 2004.

RESUMEN: El grupo étnico mapuche tiene una amplia distribución de asentamiento, predominantemente, en la zona costera de la IX Región de Chile, caracterizado por el aislamiento de centros urbanos e instituciones de la sociedad global chilena. Numerosos factores han sido considerados determinantes en una buena postura, entre ellos, los aspectos étnicos. Por tal motivo, nos propusimos identificar alteraciones a través de una evaluación postural en un grupo de individuos mapuche.

Realizamos un estudio postural en 62 individuos ( 34 mujeres y 28 hombres) pertenecientes al grupo étnico mapuche, entre 3 y 70 años de edad, de reducciones de la zona costera de la IX Región de Chile. La evaluación postural se efectuó en horas de la mañana, de acuerdo al método de Kendall.

Observamos la presencia de alteraciones a nivel de columna en el 35,5\% de los individuos adultos, acompañados de giba costal. En la región pélvica la anteversión de cadera se presentó en el 44\% de los individuos. El genu valgo se observó en el 51,6\% y el recurvatum en el $32,3 \%$ de los individuos mapuche.

El medio ambiente heterogéneo en los cuales habitan y cómo desarrollan sus actividades, podría estar influyendo en la postura de los individuos evaluados.

PALABRAS CLAVE: 1. Alteraciones posturales; 2. Escoliosis; 3. Giba costal; 4. Rodilla; 5. Grupo étnico mapuche.

\section{INTRODUCCIÓN}

El grupo étnico mapuche tiene una amplia distribución de asentamiento, bajo el sistema reduccional, en la IX Región de Chile. Aún cuando, la mayor concentración de reducciones aparece en la zona central de la provincia de Cautín, grupos importantes se ubican en la zona costera de toda la IX Región y, cada vez menores, en la zona precordillerana. Una característica relevante propia a los grupos mapuches costeros, es el aislamiento en que se encuentran con respecto a centros urbanos importantes y formas de contacto institucionalizadas de la sociedad global chilena (del Sol \& Henríquez, 1985).

La postura corporal se define como la posición de todo el cuerpo o de un segmento de éste en relación con la gravedad; o sea, es el resultado del equilibrio entre esta última y las fuerzas musculares antigravitatorias y pudiesen variar en relación a la situación en que se enfrenta (Miralles, 1998).
En la postura, pueden influir factores de tipo interno y externo. Entre los primeros, hay que considerar la información propioceptiva, cuya estimulación es fundamental para la maduración del esquema corporal, la regulación del equilibrio tónico ocular, postural y la ejecución de movimientos simples. Por otro lado, entre los factores externos encontramos los malos hábitos posturales de reposo, de trabajo y de ocio que van a determinar variaciones del centro de gravedad y de las curvaturas de la columna (Martins, 2001).

La postura se puede analizar desde los puntos de vista estático o dinámico. En estática, la postura es la posición relativa del cuerpo en el espacio donde se encuentra o de las diferentes partes del cuerpo en relación a otras. Mientras que, en sentido dinámico se define como el control minucioso de la actividad neuromuscular para mantener el centro de gravedad dentro de la base de sustentación (Simon et al., 1994).

Facultad de Medicina, Universidad de La Frontera, Temuco, Chile. 
Según Rash (1985) y Brownstein (2001) la postura en el Hombre se encuentra relacionada entre otras con: entorno social, personalidad, actitud mental, ocupación, hábito postural, genética, vestimenta, edad, nutrición, estado de salud, actividad física y modelos socioculturales.

La importancia de manejar una evaluación postural adecuada, que permita identificar hallazgos o alteraciones a distinto nivel segmentario y en distintos planos anatómicos, en un grupo étnico poco estudiado, desde el punto de vista físico, nos motivó a realizar este estudio.

\section{SUJETOS Y MÉTODO}

El estudio postural se realizó en 62 individuos (34 mujeres y 28 hombres) del grupo étnico mapuche, con edades entre 3 y 70 años, provenientes de las reducciones ubicadas en la zona costera de la IX Región de Chile, en el paralelo 38, entre los 20' y 50'.

Los individuos aceptaron voluntariamente participar de la entrevista y la evaluación postural. A cada uno de ellos, se les aplicó una ficha de evaluación postural, registrándose en ella, los hallazgos correspondientes. La evaluación postural estática, se efectuó en las primeras horas de la mañana con el paciente descubierto y en postura bípeda relajada, de acuerdo al modelo establecido por Kendall (2000).

\section{RESULTADOS}

En las 62 evaluaciones se observaron diferentes alteraciones de los parámetros posturales que se especifican a continuación.

La silueta posterior se presentó normal en el 64,5\% y desviada en el 35,5\% de los casos. Los menores de 15 años presentaron un $100 \%$ de normalidad. El $85,7 \%$ de los hombres y el $79,4 \%$ de las mujeres presentaron una columna vertebral normal.

$\mathrm{Al}$ relacionar la presencia de giba costal y tipo de escoliosis, se encontró en $22(35,5 \%)$ casos giba costal y escoliosis. De éstos, 13 (59\%) casos presentaban escoliosis tipo C; en $6(27,3 \%)$ casos escoliosis triple y en sólo $3(13,6 \%)$ casos con escoliosis tipo S.

En la evaluación de la silueta posterior se encontró: en $26(41,9 \%)$ casos normalidad en ambas rodillas, en $4(6,5 \%)$ casos con varo de rodilla y en $32(51,6 \%)$ casos con valgo de éstas.

En la evaluación del tendón calcáneo izquierdo se observó en 46 casos $(74,2 \%)$ una normalidad de éste, 14 casos $(22,6 \%)$ convergencia y sólo 2 casos $(3,2 \%)$ divergencia. Mientras que, el tendón calcáneo derecho presentó una normalidad en 49 casos (79\%), convergencia en 10 casos $(16,1 \%)$ y divergencia sólo en 3 casos $(4,8 \%)$.

En la evaluación de las escápulas se observó igual altura en el 68\%; más baja a izquierda en el 19,2\% y más baja a derecha en el 12,8\%. Escápulas aladas se observó en el $23,1 \%$ de los casos.

El triángulo de la talla se presentó: en 29 casos $(46,7 \%)$ mayor a izquierda, en 25 casos $(40,3 \%)$ mayor a derecha y en 8 casos $(12,9 \%)$ iguales.

En el 83,9\% de los casos se observó una posición de cabeza en equilibrio, el 9,7\% inclinación de ésta y sólo un $6,5 \%$ rotación.

En la evaluación de la silueta anterior se encontró simetría de tórax en el 100\% de los hombres y 85,3\% de las mujeres.

En el 20\% de los casos se presentó el parámetro de respirador bucal. En los menores de 15 años se observó este parámetro en un $41,7 \%$ mientras que en los mayores de 15 años un 58,3\%.

En la evaluación de la silueta lateral, la posición de la pelvis se observó: normalidad en 27 casos (44\%); y alteración en 35 casos (56\%). De éstos últimos: 27 casos $(44 \%)$ en anteversión, 4 casos $(6,5 \%)$ en retroversión, 3 casos (5\%) en antepulsión.

En el 90,3\% de los casos se observó normalidad en la evaluación lateral de los pies. En los menores de 15 años se presentó este parámetro en un 30,4\% mientras que en los mayores de 15 años un 69,6\%.

En la evaluación de abdomen se observó normalidad en el $26 \%$ de los hombres y sólo un $10 \%$ en mujeres.

En la evaluación de la silueta lateral se observó: normalidad en 32 casos (55\%), anteproyección de hombros en 22 casos $(35,5 \%)$ y retroproyección de hombros en 6 casos $(10 \%)$. Además, se observó recurvatum de rodilla en $20(32,3 \%)$ casos y flexum de rodilla en 10 $(16,1 \%)$ casos. 
Tabla I. Distribución porcentual de los parámetros posturales en silueta posterior de las comunidades mapuches de la zona costera de la IX Región-Chile.

\begin{tabular}{lccc}
\hline & $\begin{array}{c}\text { Igual } \\
\text { altura } \\
\%\end{array}$ & $\begin{array}{c}\text { Más baja } \\
\text { derecha } \\
\%\end{array}$ & $\begin{array}{c}\text { Más baja } \\
\text { izquierda } \\
\%\end{array}$ \\
\hline Pliegues poplíteos & 80,6 & 3,2 & 16,1 \\
Pliegues glúteos & 75,8 & 12,9 & 11,3 \\
Miembros superiores & 33,9 & 48,4 & 17,7 \\
\hline
\end{tabular}

\section{DISCUSIÓN}

Estudios sobre aspectos antropológicos físicos del grupo étnico mapuche, son muy escasos, en comparación con la gran variedad de trabajos sobre antropología social.

Mediciones de la estatura de los mapuches fueron realizados por Guevara (1899) quien indicó que ésta podía calificarse como media, (entre 170 y $140 \mathrm{~cm}$ ) en los habitantes de la zona costera y valle central, aumentando en los mapuches de las reducciones andinas. Estudios posteriores de Latchmam (1911) no arrojaron diferencias entre los mapuches de las regiones costera y cordillerana, pero sí eran de estatura más baja los habitantes de las regiones subandina y del valle central. Guevara (1927) señaló que las tallas no son de rigurosa uniformidad, por los cambios de residencia de familias y uniones matrimoniales con mujeres traidas de grupos distantes, los que han contribuido a la mezcla de estaturas en una misma zona. Esta situación se ha ido acentuando por el desplazamiento de mapuches hacia centros urbanos (del Sol \& Henríquez, 1985).

Estudios recientes sobre aspectos óseos de los individuos mapuches han sido realizados por del Sol et al., 1990; del Sol \& Olave, 1996. Por otra parte, Henríquez et al., (2003) realizaron un análisis de la estabilidad ortostática cráneo-cervical en adultos jóvenes mapuches.

La postura correcta, fundamental para el bienestar del hombre, se refiere a un proceso complejo que para llegar al equilibrio, exige de cada persona una conciencia integral de su cuerpo, de sus límites y de su localización correcta en el espacio; es decir, una profunda maduración psicosomática y personal (Martins, 2001).

Las alteraciones posturales que pueden ocurrir a lo largo del crecimiento y la maduración fisiológica, conllevan a un alineamiento incorrecto que origina estrés y una tensión innecesaria que afecta a los huesos, articulaciones, ligamentos y músculos.

Gran parte de las alteraciones observadas en este concentran a nivel de columna vertebral, pies, rodillas y pelvis. Resulta interesante resaltar que no existían alteraciones de la
Tabla II. Distribución porcentual de los parámetros posturales en silueta anterior de las comunidades mapuches de la zona costera de la IX-Región Chile.

\begin{tabular}{lccc}
\hline & $\begin{array}{c}\text { Igual } \\
\text { altura } \\
\%\end{array}$ & $\begin{array}{c}\text { Más baja } \\
\text { derecha } \\
\%\end{array}$ & $\begin{array}{c}\text { Más baja } \\
\text { izquierda } \\
\%\end{array}$ \\
\hline Patelas & 83,9 & 11,3 & 4,8 \\
Pliegues inguinales & 91,7 & 5,0 & 3,3 \\
E. I. A. S. & 86,7 & 3,3 & 10,0 \\
Hombros & 47,5 & 26,2 & 26,2 \\
\hline
\end{tabular}

silueta posterior en niños menores de 15 años, en cambio, sí observamos alteraciones en alrededor del 35\% de los individuos mayores.

La giba costal según Stokes \& Moreland (1987) se presenta con prominencia de las costillas sobre la convexidad de la curva de la columna vertebral y se debe, generalmente, a una rotación vertebral que es observada mejor en una flexión anterior de tronco. Así en nuestro estudio, en los casos donde existía una escoliosis evidente, ésta se acompañaba de giba costal. Por otra parte, Bunnel (1986) señaló que la frecuencia de escoliosis varía de acuerdo a la población estudiada, llegándose a una estimación del 2 al 4\% en la población general.

La deformidad que produce la escoliosis compromete tanto al tronco en general como a las costillas y órganos internos. Una asimetría de tórax puede llegar a ser un problema importante de salud y estética para el paciente. En este estudio se observó que las mujeres presentaban mayores alteraciones a nivel de columna vertebral (20,6\%), acompañada de asimetría de tórax en alrededor del $15 \%$ de ellas.

Sin restar importancia a la posición de los pies, que son los que establecen la base de sujeción, debe afirmarse que la posición de la pelvis representa la clave del correcto alineamiento postural, por encima o debajo de ésta ( Kendall, 2000). Se observó en nuestra investigación un elevado porcentaje de anteversión de cadera (44\%), lo que estaría incidiendo en las alteraciones de las curvaturas fisiológicas de la columna. Esto último, estaría en concordancia con lo señalado por Neto Júnior et al., (2004) quienes observaron elevados índice de anteversión de cadera $(73 \%)$ en atletas brasileños, lo que desencadenaba xifosis torácica con anteposición de cabeza. Estos investigadores indicaron que las causas de las alteraciones pélvicas estarían asociadas con mecanismos de compensación en los procesos de estabilización de la columna lumbar. Pudiese ser que, por la geografía del terreno y por las grandes distancias a recorrer, los efectos producidos a nivel de los miembros inferiores, sean una retracción de los músculos flexores de cadera y del músculo cuadríceps femoral. 
Llama la atención que en más del 50\% de los casos, existe un grado de desviación del eje normal a nivel de rodilla, siendo la alteración más frecuente el valgo y en silueta lateral el recurvatum.

En la postura de los individuos mapuche de la región costera debemos considerar factores que podrían estar influyendo en las alteraciones de los parámetros posturales evaluados, entre ellos la existencia de un medio ambiente heterogéneo, con actividades como agricultura menor, horticultura, reforestación, tala de bosques y recolección de mariscos y algas (del Sol \& Henríquez).

DEL SOL, M. \& HUNTER, K. Postural evaluation of mapuche individuals in the coastal area of the IX Region in Chile. Int. J. Morphol., 22(4):339-342, 2004.

SUMMARY: The mapuche ethnic group has an extensive settlement distribution in the coastal area of the IX Region in Chile, which is characterized by the isolation from urban centers and institutions of the global Chilean society. As ethnic features are considered one of the numerous factors that influence good body posture, we decided to carry out this investigation.

The postural exam was performed on 62 individuals ( 34 women and 28 men) between the ages of three and seventy, belonging to the Mapuche ethnic group, from parts of the costal area of the IX region in Chile. The postural evaluation took place during the morning hours, according to the Kendall method.

We observed alterations at the level of the spine in $33.5 \%$ of adult individuals, accompanied by a costal hump. In the pelvic region the anterior version of the hip was present in $44 \%$ of the individuals. The genu valgo was observed in $51.6 \%$ and the recurvatum in $32.3 \%$ of the individuals.

The heterogeneous environment where they live, their customs and additional social and cultural factors, could have an influence in the posture of the evaluated.

KEY WORDS: 1. Postural alterations; 2. Scoliosis; 3. Costal hump; 4. Knee; 5. mapuche ethnic group.

\section{REFERENCIAS BIBLIOGRÁFICAS}

Adams, M. \& Hutton,W. The effect of posture on the lumbar spine. $J$. Bone Joint Surgery, 67B:625-9, 1985.

Aravena, G.; Pinochet, A. \& Fuentes, J. Alteraciones posturales en niños escolares de la ciudad de Talca de distinto nivel socioeconómico. Revista Chilena Kinesiología, 74:6-12, 2004.

Brownstein, A. La curación natural de la espalda. Barcelona, Paidotribo, 2001.

Del Sol, M. \& Henríquez, J. Evaluación de algunos índices antropométricos de un grupo de población mapuche. Bol. Mus. Reg. Araucanía, 2:79:89, 1985 .

Del Sol, M.; Vieira, M. C. \& Olave, E. Estudo morfométrico do esterno no grupo étnico Mapuche. Arq. Anat. Antropol., 41:211-6, 1990.

Del Sol, M. \& Olave, E. Índices claviculares en el grupo étnico mapuche. Rev. Chil. Anat., 14(2):183-88, 1996.

Gagey, P. M. \& Weber, B. Posturología: Regulación y alteraciones de la bipedestación. Barcelona, Masson, 2001.

Guevara, T. Etnografía araucana. In Historia de la civilización de la araucanía. Ann. Univ. de Chile, 103:306-17, 1899.

Henríquez, J.; Fuentes, R.; Sandoval, P. \& Muñoz, A. Análisis de la estabilidad ortostática cráneocervical en adultos jóvenes mapuches. Int. J. Morphol., 21(2):149-53, 2003.

Igual, C.; Muñoz, E. \& Aramburu, C. Fisioterapia General: Cinesiterapia. Madrid, Síntesis, 1996. V. 3.
Kendall, E.; Peterson, F. \& Geise, P. Músculos: Pruebas, funciones y dolor postural. $4^{\mathrm{a}}$. ed. Madrid, Marban, 2000.

Latchmam, R. Antropología chilena. In primer Congreso Científico Panamericano, Santiago de Chile. Secc. $3^{\circ}$. Ciencias Naturales, Antropología y Etnografía, 1:24-84, 1911.

Miralles, R. Biomecánica clínica del aparato locomotor. Barcelona, Masson, 1998.

Neto Júnior, J.; Pastro, C. M. \& Monteiro, L. H. Alterações posturais em atletas brasileiros do sexo masculino que participaram de provas de potência muscular em competições internacionais. Rev. Bras. med. Esporte, 10(3):195-8, 2004.

Palmer, M \& Epler, M. Fundamentals of musculoskeletal assessment techniques. 2a. ed. New York, Lippincott, 1998.

Rash, P.; Burke, R. Kinesiología y anatomía aplicada. 6a. ed. Barcelona, $\mathrm{El}$ ateneo, 1985.

Dirección para correspondencia

Prof. Karina Hunter Echeverría

Facultad de Medicina

Universidad de La F Fontrea

Casilla 54-D

Temuco-Chile

Recibido : 12-07-2004

Email:Kari_funterkine@yahoo.com_Aceptado: 24-10-2004 